\title{
A Kind of Complete Moment Convergence for Sums of Independent and Nonidentically Distributed Random Variables
}

\author{
Bao Wang ${ }^{1}$ and Quanxin $\mathrm{Zhu}^{2}$ \\ ${ }^{1}$ College of Mathematics and Physics, Xuzhou Institute of Technology, Xuzhou 221000, China \\ ${ }^{2}$ School of Mathematical Sciences and Institute of Finance and Statistics, Nanjing Normal University, Nanjing, Jiangsu 210023, China \\ Correspondence should be addressed to Quanxin Zhu; zqx22@126.com
}

Received 21 June 2014; Accepted 26 August 2014; Published 14 October 2014

Academic Editor: Jesús Marín-Solano

Copyright (c) 2014 B. Wang and Q. Zhu. This is an open access article distributed under the Creative Commons Attribution License, which permits unrestricted use, distribution, and reproduction in any medium, provided the original work is properly cited.

Let $\left\{X, X_{n}, n \geq 1\right\}$ be a sequence of independent and nonidentically distributed random variables. We obtain a new kind of complete moment convergence for their sums under the Lyapunov condition. Moreover, our result extends and improves the corresponding result of the independent and identically distributed (i.i.d.) cases.

\section{Introduction and Main Result}

Let $\left\{X, X_{n}, n \geq 1\right\}$ be a sequence of random variables, and $S_{n}=\sum_{k=1}^{n} X_{k}$. If for every $\varepsilon>0, \sum_{n=1}^{+\infty} P\left\{\left|X_{n}\right|>\varepsilon\right\}<\infty$, then $\left\{X, X_{n}, n \geq 1\right\}$ is said to converge to 0 completely.

Hsu and Robbins [1] proved that if $\left\{X, X_{n}, n \geq 1\right\}$ is a sequence of independent and identically distributed (i.i.d.) random variables with $E X_{1}=\mu$, and $E X_{1}^{2}<\infty$, then $S_{n} / n \rightarrow$ $\mu$ completely.

Erdos $[2,3]$ proved that if $\left\{X, X_{n}, n \geq 1\right\}$ is a sequence of i.i.d. random variables, then for every $\varepsilon>0, \sum_{n=1}^{+\infty} P\left\{\left|S_{n}\right| / n>\right.$ $\varepsilon\}<\infty$ holds if and only if $E X_{1}=\mu$ and $E X_{1}^{2}<\infty$.

Obviously the sum tends to infinity as $\varepsilon \downarrow 0$, and it is necessary to study the convergence rate in which this occurs; Heyde [4] proved that

$$
\lim _{\varepsilon \downarrow 0} \varepsilon^{2} \sum_{n=1}^{+\infty} P\left(\left|S_{n}\right| \geq \varepsilon n\right)=E X^{2},
$$

when $E X=0$ and $E X^{2}<\infty$. This research direction is known as the precise asymptotics. For analogous results in more general case, we refer the reader to [5-14] and the references therein.

Recently, Liu and Lin [15] have introduced a new kind of complete moment convergence and obtained the following result.
Theorem A (see [15]). Suppose that $\left\{X, X_{n}, n \geq 1\right\}$ is a sequence of independent and identically distributed (i.i.d.) random variables. Then

$$
\lim _{\varepsilon \downarrow 0} \frac{1}{-\log \varepsilon} \sum_{n=1}^{\infty} \frac{1}{n^{2}} E\left[S_{n}^{2} I\left\{\left|S_{n}\right| \geq \varepsilon n\right\}\right]=2 \sigma^{2}
$$

holds if and only if $E X_{k}=0, E\left[X_{k}^{2}\right]=\sigma^{2}$, and $E\left[X_{k}^{2} \log ^{+}\left|X_{k}\right|\right]<\infty$, where $k \in Z$ and $k \geq 1$.

However, the condition of identical distribution is very strong and rather difficult to verify in some real cases. The following theorem gives a sufficient condition of complete moment convergence for independent nonidentically distributed random variables.

Theorem 1. Let $\left\{X, X_{n}, n \geq 1\right\}$ be independent random variables such that $E X_{n}=0$ and $E\left[X_{n}^{2}\right]=\sigma_{n}^{2}<\infty, n \in N$. Assume that there exists a constant c such that $\left|X_{k}\right| \leq c B_{n}$, a.s., where $B_{n}^{2}=\sum_{k=1}^{n} \sigma_{k}^{2}$. Moreover, one also assumes that the following Lyapunov condition [16, page 298] is satisfied:

$$
\lim _{n \rightarrow+\infty} B_{n}^{-2-\delta} \sum_{j=1}^{n} E\left|X_{j}\right|^{2+\delta}=0
$$


where $0<\delta \leq 1$. Then, one has

$$
\lim _{\varepsilon \downarrow 0} \frac{1}{-\log \varepsilon} \sum_{n=1}^{+\infty} \frac{1}{n} E\left[\frac{S_{n}^{2}}{B_{n}^{2}} I_{\left\{\left|S_{n}\right| / B_{n} \geq \varepsilon \sqrt{n}\right\}}\right]=2 .
$$

Remark 2. Suppose that $\left\{X, X_{n}, n \geq 1\right\}$ is a sequence of independent and identically distributed (i.i.d.) random variables with $E\left[\left|X_{n}^{2+\delta}\right|\right]<\infty$, where $0<\delta \leq 1$ is a constant. It is easy to verify the Lyapunov condition (3) in real applications, and so the Lyapunov condition (3) is much weaker than the identically distributed condition. Moreover, the Lyapunov condition (3) constrains the growth rate of moment.

Many sequences of independent random variables satisfy Lyapunov's condition; here we give some examples.

Example 1. Let $\left\{X, X_{n}, n \geq 1\right\}$ be a sequence of independent random variables satisfying $E X_{k}=\mu_{k}, \operatorname{Var} X_{k}=\sigma_{k}^{2}, k \geq 1$, and $B_{n}^{2} \rightarrow \infty(n \rightarrow \infty)$. Suppose that $X_{n}, n \geq 1$ are uniformly bounded; that is, there exists a constant $M$ such that $\left|X_{n}\right| \leq M$ for all $n \geq 1$, and then we have

$$
\begin{gathered}
\sum_{k=1}^{n} E\left|X_{k}-\mu_{k}\right|^{2+1} \leq 2 M \sum_{k=1}^{n} E\left|X_{k}-\mu_{k}\right|^{2}=2 M B_{n}^{2}, \\
\lim _{n \rightarrow+\infty} B_{n}^{-2-1} \sum_{j=1}^{n} E\left|X_{j}\right|^{2+1} \leq \lim _{n \rightarrow+\infty} \frac{2 M B_{n}^{2}}{B_{n}^{2+1}}=2 M \lim _{n \rightarrow+\infty} \frac{1}{B_{n}}=0,
\end{gathered}
$$

which verifies that $\left\{X, X_{n}, n \geq 1\right\}$ satisfies the Lyapunov condition (3).

Example 2. Let $\left\{X, X_{n}, n \geq 1\right\}$ be a sequence of independent random variables, which satisfies $P\left(X_{n}=1\right)=p_{n}, P\left(X_{n}=\right.$ $0)=1-p_{n}$, and

$$
p_{n}= \begin{cases}\frac{1}{2}, & n=2 k, k \in N, \\ \frac{1}{3}, & n=2 k+1, k \in N .\end{cases}
$$

By Example 1, we know that $\left\{X, X_{n}, n \geq 1\right\}$ satisfies the Lyapunov condition (3).

Remark 3. Suppose that $\left\{X, X_{n}, n \geq 1\right\}$ is a sequence of independent and identically distributed (i.i.d.) random variables such that $E X_{n}=0, E\left[X_{n}^{2}\right]=\sigma^{2}$ for all $n \geq 1$, $E\left[\left|X_{n}^{2+\delta}\right|\right]<\infty$, where $\delta>0$ is a constant. Then, from Remark 2, we know that it satisfies Lyapunov's condition. Therefore, by Theorem 1, we have

$$
\lim _{\varepsilon \downarrow 0} \frac{1}{-\log \varepsilon} \sum_{n=1}^{\infty} \frac{1}{n^{2}} E\left[S_{n}^{2} I\left\{\left|S_{n}\right| \geq \varepsilon n\right\}\right]=2 \sigma^{2} .
$$

Obviously, this case is the result of Liu and Lin [15]. Therefore, our condition of Theorem 1 is different from the conditions of Theorem A, and our result partly extends and improves those given in Liu and Lin [15].

\section{Proof of Theorem 1}

In this section, we will prove Theorem 1 . We first present the following two lemmas, which play a key role in the proof of Theorem 1 .

Lemma 4 (see [17]). Suppose that $\left\{X, X_{n}, n \geq 1\right\}$ are independent random variables with $E X_{n}=0$ and $E\left[X_{n}^{2}\right]=$ $\sigma_{n}^{2}<\infty$, where $n \in N$. Let $B_{n}^{2}=\sum_{j=1}^{n} \sigma_{j}^{2}, F_{n}(x)=$ $P\left(\left(\sum_{j=1}^{n} X_{j}\right) / B_{n} \leq x\right)$, and $\Delta_{n}(x)=\left|F_{n}(x)-\Phi(x)\right|$, where $\Phi(x)$ is the standard normal distribution function. If $E\left|X_{j}\right|^{2+\delta}<\infty$, $j=1,2, \ldots, n$, for some $0 \leq \delta \leq 1$, then for every $x$,

$$
\Delta_{n}(x) \leq A B_{n}^{-2-\delta} \sum_{j=1}^{n} E\left|X_{j}\right|^{2+\delta}\left(1+|x|^{2+\delta}\right)^{-1}
$$

holds.

Lemma 5 (see page 73 of [18]). Under the conditions of Lemma 4, if $\left|X_{j}\right| \leq c B_{n}$ a.s., $j=1,2, \ldots, n$, where $c>0$, then for every $x>0$,

$$
P\left(\frac{\left|S_{n}\right|}{B_{n}} \geq x\right) \leq 2 \exp \left(-\frac{x}{2 c} \sinh \left(\frac{x c}{2}\right)\right) .
$$

Proof of Theorem 1. Similar to [15], we have

$$
\begin{aligned}
\sum_{n=1}^{+\infty} & \frac{1}{n} E\left[\frac{S_{n}^{2}}{B_{n}^{2}} I\left\{\frac{\left|S_{n}\right|}{B_{n}} \geq \varepsilon \sqrt{n}\right\}\right] \\
\quad= & \varepsilon^{2} \sum_{n=1}^{+\infty} P\left(\frac{\left|S_{n}\right|}{B_{n}} \geq \varepsilon \sqrt{n}\right)+\sum_{n=1}^{+\infty} \frac{1}{n} \int_{\varepsilon \sqrt{n}}^{+\infty} 2 t P\left(\frac{\left|S_{n}\right|}{B_{n}} \geq t\right) d t \\
& :=I_{1}+I_{2} .
\end{aligned}
$$

To prove Theorem 1 , we only need to study $I_{1}$ and $I_{2}$. We will divide the proof into two steps.

Step 1. We first prove the equality as follows:

$$
\lim _{\varepsilon \downarrow 0} \frac{1}{-\log \varepsilon} \varepsilon^{2} \sum_{n=1}^{+\infty} P\left(\frac{\left|S_{n}\right|}{B_{n}} \geq \varepsilon \sqrt{n}\right)=0 .
$$

In fact, it follows from Proposition 2.1.1 of [19] that

$$
\lim _{\varepsilon \downarrow 0} \varepsilon^{2} \sum_{n=1}^{+\infty} P(|N| \geq \varepsilon \sqrt{n})=1 .
$$

By (12), we obtain

$$
\lim _{\varepsilon \downarrow 0} \frac{1}{-\log \varepsilon} \varepsilon^{2} \sum_{n=1}^{+\infty} P(|N| \geq \varepsilon \sqrt{n})=0 .
$$

To establish the equality (11), from (13) we only need to prove that

$$
\lim _{\varepsilon \downarrow 0} \frac{1}{-\log \varepsilon} \varepsilon^{2} \sum_{n=1}^{+\infty}\left|P\left(\frac{\left|S_{n}\right|}{B_{n}} \geq \varepsilon \sqrt{n}\right)-P(|N| \geq \varepsilon \sqrt{n})\right|=0 .
$$


Obviously, it follows from Lemma 4 that

$$
\begin{aligned}
& \left|P\left(\frac{\left|S_{n}\right|}{B_{n}} \geq \varepsilon \sqrt{n}\right)-P(|N| \geq \varepsilon \sqrt{n})\right| \\
& \quad=2 \Delta_{n}(\varepsilon \sqrt{n}) \\
& \quad \leq 2 A B_{n}^{-2-\delta}\left(1+|\varepsilon \sqrt{n}|^{2+\delta}\right)^{-1} \sum_{j=1}^{n} E\left|X_{j}\right|^{2+\delta} .
\end{aligned}
$$

Combining (3) and (15), we get

$$
\lim _{n \rightarrow+\infty}\left|P\left(\frac{\left|S_{n}\right|}{B_{n}} \geq \varepsilon \sqrt{n}\right)-P(|N| \geq \varepsilon \sqrt{n})\right|=0 .
$$

Since

$$
\lim _{\varepsilon \downarrow 0} \sum_{n=1}^{\left[1 / \varepsilon^{2}\right]} \varepsilon^{2} \leq 1,
$$

it follows from Toeplitz's lemma (page 120 of [20]) that

$$
\lim _{\varepsilon \downarrow 0} \varepsilon^{2} \sum_{n=1}^{\left[1 / \varepsilon^{2}\right]}\left|P\left(\frac{\left|S_{n}\right|}{B_{n}} \geq \varepsilon \sqrt{n}\right)-P(|N| \geq \varepsilon \sqrt{n}]\right|=0 .
$$

By (18), we have

$$
\lim _{\varepsilon \downarrow 0} \frac{1}{-\log \varepsilon} \varepsilon^{2} \sum_{n=1}^{\left[1 / \varepsilon^{2}\right]}\left|P\left(\frac{\left|S_{n}\right|}{B_{n}} \geq \varepsilon \sqrt{n}\right)-P(|N| \geq \varepsilon \sqrt{n}]\right|=0 .
$$

On the other hand, it follows from Lemma 5 that

$$
\begin{aligned}
& \varepsilon^{2} \sum_{n \geq\left[1 / \varepsilon^{2}\right]} P\left(\frac{\left|S_{n}\right|}{B_{n}} \geq \varepsilon \sqrt{n}\right) \\
& \quad \leq 2 \varepsilon^{2} \int_{1 / \varepsilon^{2}}^{+\infty} \exp \left(-\frac{\varepsilon \sqrt{t}}{2 c} \sinh \left(\frac{\varepsilon \sqrt{t} c}{2}\right)\right) d t \\
& =2 \varepsilon^{2} \int_{1}^{+\infty} \exp \left(-\frac{u}{2 c} \sinh \left(\frac{u c}{2}\right)\right) \frac{2 u}{\varepsilon^{2}} d t \\
& =4 \int_{1}^{+\infty} u \exp \left(-\frac{u}{2 c} \sinh \left(\frac{u c}{2}\right)\right) d u .
\end{aligned}
$$

Noting that

$$
\int_{1}^{+\infty} u \exp \left(-\frac{u}{2 c} \sinh \left(\frac{u c}{2}\right)\right) d u<\infty
$$

the inequality (20) yields

$$
\varepsilon^{2} \sum_{n \geq\left[1 / \varepsilon^{2}\right]} P\left(\frac{\left|S_{n}\right|}{B_{n}} \geq \varepsilon \sqrt{n}\right)<\infty
$$

By (22), we obtain

$$
\lim _{\varepsilon \downarrow 0} \frac{1}{-\log \varepsilon} \varepsilon^{2} \sum_{\left[1 / \varepsilon^{2}\right]}^{+\infty}\left|P\left(\frac{\left|S_{n}\right|}{B_{n}} \geq \varepsilon \sqrt{n}\right)-P(|N| \geq \varepsilon \sqrt{n})\right|=0 .
$$

Combining (19) and (23), we see that the equality (11) is satisfied.

Step 2. Next, we need to prove the following equality:

$$
\lim _{\varepsilon \downarrow 0} \frac{1}{-\log \varepsilon} \sum_{n=1}^{+\infty} \frac{1}{n} \int_{\varepsilon \sqrt{n}}^{+\infty} 2 t P\left(\frac{\left|S_{n}\right|}{B_{n}} \geq t\right) d t=2 .
$$

Obviously, it follows from Proposition 3.1 of [15] that

$$
\lim _{\varepsilon \downarrow 0} \frac{1}{-\log \varepsilon} \sum_{n=1}^{+\infty} \frac{1}{n} \int_{\varepsilon \sqrt{n}}^{+\infty} 2 t P(|N| \geq t) d t=2 .
$$

To establish (24), from (25) we only need to prove

$$
\lim _{\varepsilon \downarrow 0} \frac{1}{-\log \varepsilon} \sum_{n=1}^{+\infty} \frac{1}{n} \int_{\varepsilon \sqrt{n}}^{+\infty} 2 t\left|P\left(\frac{\left|S_{n}\right|}{B_{n}} \geq t\right)-P(|N| \geq t)\right| d t=0 .
$$

Letting $x=t / \sqrt{n}-\varepsilon$ and $L_{n}=\left(A \sum_{j=1}^{n} E\left|X_{j}\right|^{2+\delta}\right) / B_{n}^{2+\delta}$, we apply Lemma 4 to obtain

$$
\begin{aligned}
& \int_{\varepsilon \sqrt{n}}^{+\infty} 2 t\left|P\left(\frac{\left|S_{n}\right|}{B_{n}} \geq t\right)-P(|N| \geq t)\right| d t \\
& =\int_{0}^{+\infty} 2 \sqrt{n}(x+\varepsilon) \mid P\left(\frac{\left|S_{n}\right|}{B_{n}} \geq \sqrt{n}(x+\varepsilon)\right) \\
& \quad-P(|N| \geq \sqrt{n}(x+\varepsilon)) \mid \sqrt{n} d x \\
& =2 \int_{0}^{+\infty} n(x+\varepsilon) 2 \Delta_{n}(\sqrt{n}(x+\varepsilon)) d x \\
& \leq 2 \int_{0}^{+\infty} n(x+\varepsilon) L_{n} \frac{1}{1+[\sqrt{n}(x+\varepsilon)]^{2+\delta}} d x . \\
& \text { If } n \leq\left[1 / \varepsilon^{2}\right] \text {, then it follows from (3) that }
\end{aligned}
$$

$$
\begin{aligned}
& \int_{0}^{1 / \sqrt{n}} n(x+\varepsilon) L_{n} \frac{1}{1+[\sqrt{n}(x+\varepsilon)]^{2+\delta}} d x \\
& \quad \leq \int_{0}^{1 / \sqrt{n}} n(x+\varepsilon) L_{n} d x \\
& \quad=\left.\frac{1}{2} n L_{n}(x+\varepsilon)^{2}\right|_{0} ^{1 / \sqrt{n}} \leq \frac{3}{2} L_{n} \longrightarrow 0 \quad(n \longrightarrow \infty),
\end{aligned}
$$

$$
\begin{gathered}
\int_{1 / \sqrt{n}}^{1} n(x+\varepsilon) L_{n} \frac{1}{1+[\sqrt{n}(x+\varepsilon)]^{2+\delta}} d x \\
\quad \leq \int_{1 / \sqrt{n}}^{1} n^{-\delta / 2}(x+\varepsilon)^{-1-\delta} L_{n} d x
\end{gathered}
$$




$$
\begin{aligned}
& \quad \int_{1 / \sqrt{n}}^{1} n^{-\delta / 2} x^{-1-\delta} L_{n} d x \\
& =\left.L_{n} n^{-\delta / 2}\left[-\frac{1}{\delta} x^{-\delta}\right]\right|_{1 / \sqrt{n}} ^{1} \leq \frac{1}{\delta} L_{n} \rightarrow 0 \quad(n \longrightarrow \infty), \\
& \int_{1}^{+\infty} n(x+\varepsilon) \frac{1}{n^{1+\delta / 2}(x+\varepsilon)^{2+\delta}} d x \\
& =n^{-\delta / 2} \int_{1}^{+\infty}(x+\varepsilon)^{-1-\delta} d x \rightarrow 0 \quad(n \rightarrow \infty) .
\end{aligned}
$$

Hence, by (27) and (28), we have that for $n \leq\left[1 / \varepsilon^{2}\right]$, the following holds:

$$
\int_{\varepsilon \sqrt{n}}^{+\infty} 2 t\left|P\left(\frac{\left|S_{n}\right|}{B_{n}} \geq t\right)-P(|N| \geq t)\right| d t \rightarrow 0 \quad(n \longrightarrow \infty) \text {. }
$$

Noting the fact that the weighted average of a sequence that converge to 0 also converges to 0 , we have

$$
\begin{array}{r}
\sum_{n=1}^{\left[1 / \varepsilon^{2}\right]} \frac{1}{n} \int_{\varepsilon \sqrt{n}}^{+\infty} 2 t\left|P\left(\frac{\left|S_{n}\right|}{B_{n}} \geq t\right)-P(|N| \geq t)\right| d t \\
\text { as } \varepsilon \longrightarrow 0
\end{array}
$$

and so

$$
\begin{aligned}
& \lim _{\varepsilon \downarrow 0} \frac{1}{-\log \varepsilon} \sum_{n=1}^{\left[1 / \varepsilon^{2}\right]} \frac{1}{n} \int_{\varepsilon \sqrt{n}}^{+\infty} 2 t\left|P\left(\frac{\left|S_{n}\right|}{B_{n}} \geq t\right)-P(|N| \geq t)\right| d t \\
& \quad=0 .
\end{aligned}
$$

If $n \geq\left[1 / \varepsilon^{2}\right]$, it follows from (8) and (3) that

$$
\begin{aligned}
& \int_{\varepsilon \sqrt{n}}^{+\infty} 2 t\left|P\left(\frac{\left|S_{n}\right|}{B_{n}} \geq t\right)-P(|N| \geq t)\right| d t \\
& \quad \leq \int_{1}^{+\infty} 4 t A B_{n}^{-2-\delta}\left(1+|t|^{2+\delta}\right)^{-1} \sum_{j=1}^{n} E\left|X_{j}\right|^{2+\delta} d t \\
& \quad \leq 4 L_{n} \int_{1}^{+\infty} \frac{1}{t^{1+\delta}} d t \longrightarrow 0 \quad(n \longrightarrow \infty) .
\end{aligned}
$$

Obviously, by (32), we get

$$
\begin{aligned}
& \lim _{\varepsilon \downarrow 0} \frac{1}{-\log \varepsilon} \sum_{n=\left[1 / \varepsilon^{2}\right]}^{+\infty} \frac{1}{n} \int_{\varepsilon \sqrt{n}}^{+\infty} 2 t\left|P\left(\frac{\left|S_{n}\right|}{B_{n}} \geq t\right)-P(|N| \geq t)\right| d t \\
& \quad=0 .
\end{aligned}
$$

Combining (31) and (33), we have

$$
\lim _{\varepsilon \downarrow 0} \frac{1}{-\log \varepsilon} \sum_{n=1}^{+\infty} \frac{1}{n} \int_{\varepsilon \sqrt{n}}^{+\infty} 2 t\left|P\left(\frac{\left|S_{n}\right|}{B_{n}} \geq t\right)-P(|N| \geq t)\right| d t=0,
$$

which implies that (24) is satisfied.
Therefore, from (11) and (34), we see that (4) is true. This completes the proof of Theorem 1 .

\section{Conflict of Interests}

The authors declare that they have no competing interests.

\section{Acknowledgments}

The authors are thankful to the referees for their many valuable suggestions. This work was jointly supported by the National Natural Science Foundation of China (61374080), The Natural Science Foundation for Colleges and Universities of Jiangsu Province (14KJB110025), the Natural Science Foundation of Zhejiang Province (LY12F03010), the Natural Science Foundation of Ningbo (2012A610032), the Postgraduate Innovation Projection of Jiangsu University (CXLX12-0652), Youth Foundation of Xuzhou Institute of Technology (XKY2012301), and a Project Funded by the Priority Academic Program Development of Jiangsu Higher Education Institutions.

\section{References}

[1] P. L. Hsu and H. Robbins, "Complete convergence and the law of large numbers," Proceedings of the National Academy of Sciences of the United States of America, vol. 33, pp. 25-31, 1947.

[2] P. Erdos, "On a theorem of Hsu and Robbins," Annals of Mathematical Statistics, vol. 20, pp. 286-291, 1949.

[3] P. Erdos, "Remark on my paper "On a theorem of Hsu and Robbins'”' The Annals of Mathematical Statistics, vol. 21, no. 1, p. 138, 1950.

[4] C. C. Heyde, "A supplement to the strong law of large numbers," Journal of Applied Probability, vol. 12, pp. 173-175, 1975.

[5] R. Chen, "A remark on the tail probability of a distribution," Journal of Multivariate Analysis, vol. 8, no. 2, pp. 328-333, 1978.

[6] A. Gut and A. Spătaru, "Precise asymptotics in the BaumKatz and Davis laws of large numbers," Journal of Mathematical Analysis and Applications, vol. 248, no. 1, pp. 233-246, 2000.

[7] A. Gut and A. Spătaru, "Precise asymptotics in the law of the iterated logarithm," The Annals of Probability, vol. 28, no. 4, pp. 1870-1883, 2000.

[8] A. Gut and A. Spătaru, "Precise asymptotics in some strong limit theorems for multidimensionally indexed random variables," Journal of Multivariate Analysis, vol. 86, no. 2, pp. 398-422, 2003.

[9] H. Lanzinger and U. Stadtmüller, "Refined Baum-Katz laws for weighted sums of iid random variables," Statistics \& Probability Letters, vol. 69, no. 3, pp. 357-368, 2004.

[10] A. Spătaru, "Exact asymptotics in log log laws for random fields," Journal of Theoretical Probability, vol. 17, no. 4, pp. 943965, 2004.

[11] A. Spataru, "Precise asymptotics for a series of T. L. lai," Proceedings of the American Mathematical Society, vol. 132, no. 11, pp. 3387-3395, 2004.

[12] W. Huang and L. Zhang, "Precise rates in the law of the logarithm in the Hilbert space," Journal of Mathematical Analysis and Applications, vol. 304, no. 2, pp. 734-758, 2005. 
[13] Y.S. Chow, "On the rate of moment convergence of sample sums and extremes," Bulletin of the Institute of Mathematics. Academia Sinica, vol. 16, no. 3, pp. 177-201, 1988.

[14] Y. Jiang and L. X. Zhang, "Precise rates in the law of iterated logarithm for the moment of I.I.D. random variables," Acta Mathematica Sinica, vol. 22, no. 3, pp. 781-792, 2006.

[15] W. Liu and Z. Lin, "Precise asymptotics for a new kind of complete moment convergence," Statistics \& Probability Letters, vol. 76, no. 16, pp. 1787-1799, 2006.

[16] Y. S. Chow and H. Teicher, Probability Theory: Independence, Interchangeability, Martingales, Springer, 2nd edition, 1988.

[17] A. Bikjalis, "Estimates of the remainder term in the central limit theorem," Lietuvos Matematikos Rinkinys, vol. 6, pp. 323-346, 1966.

[18] Z. Y. Lin and Z. D. Bai, Probability Inequalities, Science Press, Beijing, China, 2006, (Chinese).

[19] Q. Zang, "A kind of complete moment convergence for selfnormalized sums," Computers \& Mathematics with Applications, vol. 60, no. 6, pp. 1803-1809, 2010.

[20] W. F. Stout, Almost Sure Convergence, Academic Pres, New York, NY, USA, 1974. 


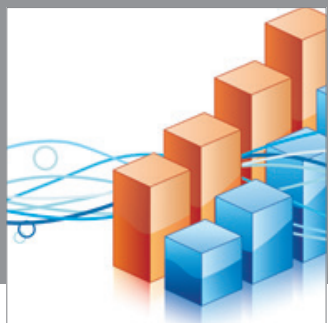

Advances in

Operations Research

mansans

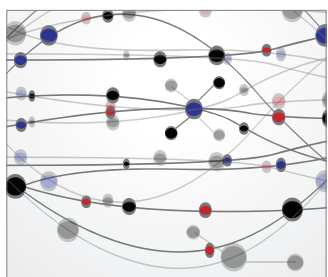

The Scientific World Journal
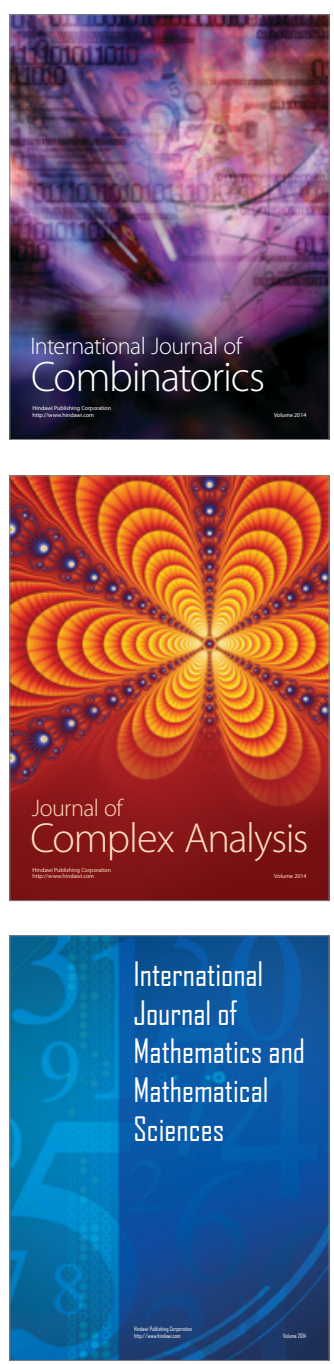
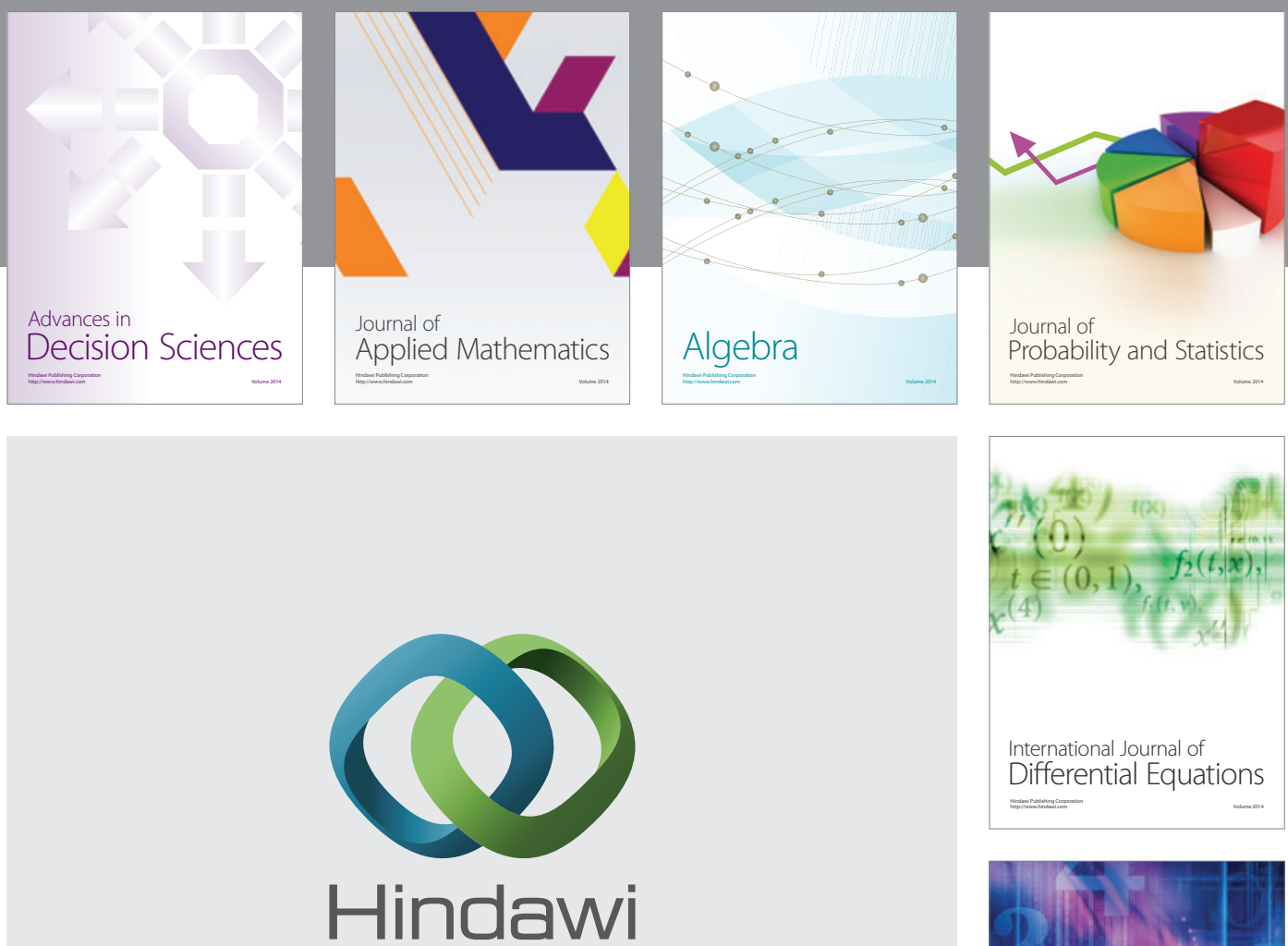

Submit your manuscripts at http://www.hindawi.com
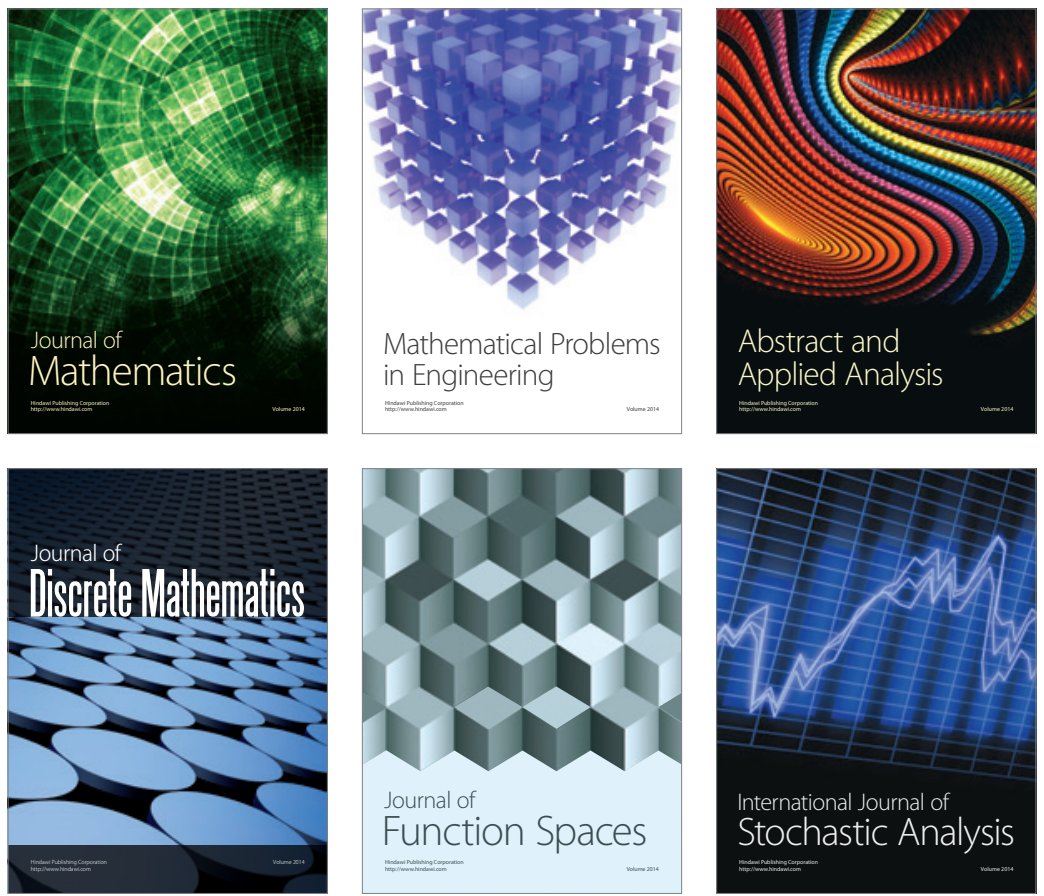

Journal of

Function Spaces

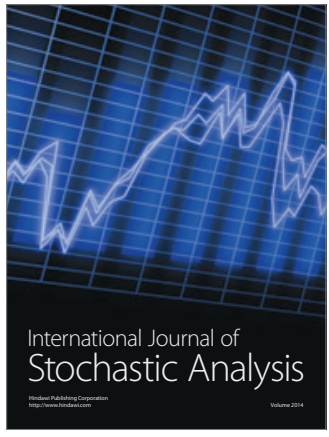

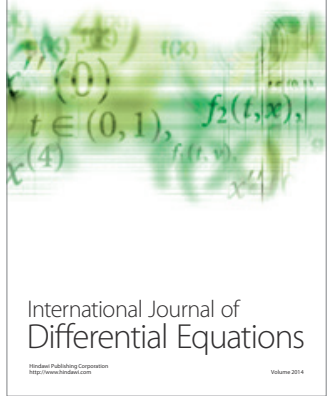
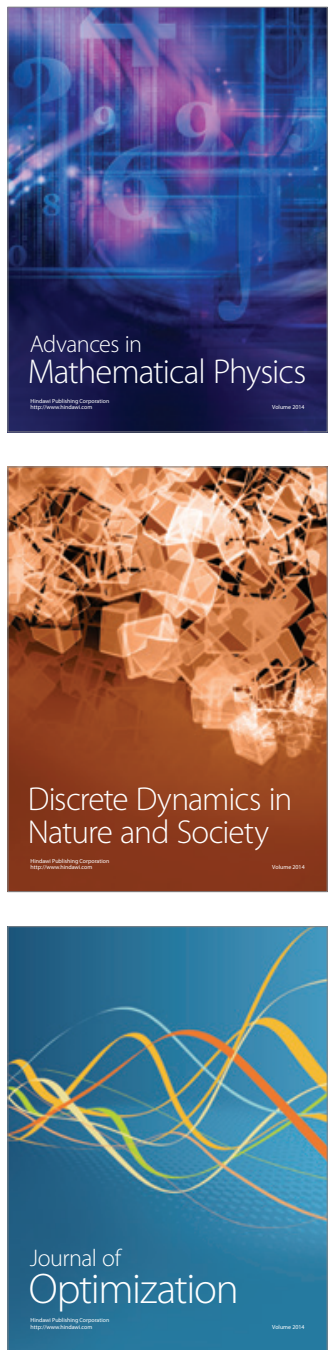